\title{
Hard core attraction in hadron scattering and the family of the Ds meson molecule
}

\author{
Pedro Bicudo* and Gonçalo M. Marques* \\ ${ }^{*}$ Dep. Física and CFIF, Instituto Superior Técnico, Av. Rovisco Pais 1049-001 Lisboa, Portugal
}

\begin{abstract}
We study the discovered Ds(2317) at BABAR, CLEO and BELLE, and find that it belongs to a class of strange multiquarks, which is equivalent to the class of kaonic molecules bound by hard core attraction. In this class of hadrons a kaon is trapped by a s-wave meson or baryon. To describe this class of multiquarks we apply the Resonating Group Method, and extract the hard core kaon-meson(baryon)interactions. We derive a criterion to classify the attractive channels. We find that the mesons f0(980), Ds(2457), Bs scalar and axial, and also the baryons with the quantum numbers of $\Lambda, \Xi_{c}, \Xi_{b}$ and also $\Omega_{c c}, \Omega_{c b}$ and $\Omega_{b b}$ belong to the new hadronic class of the Ds(2317).
\end{abstract}

Recently new narrow scalar resonances $D_{S}(2317)$ and $D_{s}(2457)$ were discovered at BABAR [1], CLEO [2] and BELLE [3]. These positive parity resonances [4] were also predicted by Nowak, Ro and Zahed [5, 6] as chiral partners of the well known negative parity $D_{s}(1968)$ and $D_{s}(2112)$. Here we find that the new $D_{s}$ resonances can be undesrtood as tetraquarks, or equivalently as $D-K$ molecules [7] bound by the hardcore attraction. These are not standard hadrons because they are neither quarkantiquark mesons nor three quark baryons. The experimental discovery of these hadrons also prompts us to study the new hadronic class which includes the $D_{s}(2317)$. In our framework the masses and couplings of hadrons are microscopically computed at the quark level and in a chiral invariant framework, [8, 9, 10].

In this talk we study the family of all possible narrow tetraquark and pentaquark resonances [11] where the quark $s$, or the Kaon play a crucial role. We start by reviewing the Resonating Group Method (RGM) [12]. The RGM, together with chiral symmetry, produces hard core hadron-hadron potentials, which can be either repulsive or attractive. We derive a criterion to discriminate which systems bind and which are unbound. We apply this criterion to find, among the s-wave hadrons, the candidates to trap a kaon. Finally we compute the binding energy of the selected hadrons, the positive parity mesons $f_{0}(980), D_{s}^{(0+)}, D_{s}^{(1+)}, B_{s}^{(0+)}, B_{s}^{(1+)}$ and the negative parity baryons $\Lambda(1405), \Xi_{c}^{(-)}, \Xi_{b}^{(-)}$ and also $\Omega_{c c}^{(-)}, \Omega_{c b}^{(-)}$and $\Omega_{b b}^{(-)}$.

The RGM computes the effective multiquark energy using the matrix elements of the microscopic quark-quark interactions. The multiquark state is decomposed in antisymmetrized combinations of simpler colour singlets, the baryons and mesons. The RGM was first used in hadronic physics by Ribeiro [13] to show that in exotic hadronhadron scattering, the quark-quark potential together with the Pauli repulsion of quarks produces a repulsive short range interaction. For instance this explains the $N-N$ hard core repulsion, preventing nuclear collapse. Deus and Ribeiro [14] used the same RGM 

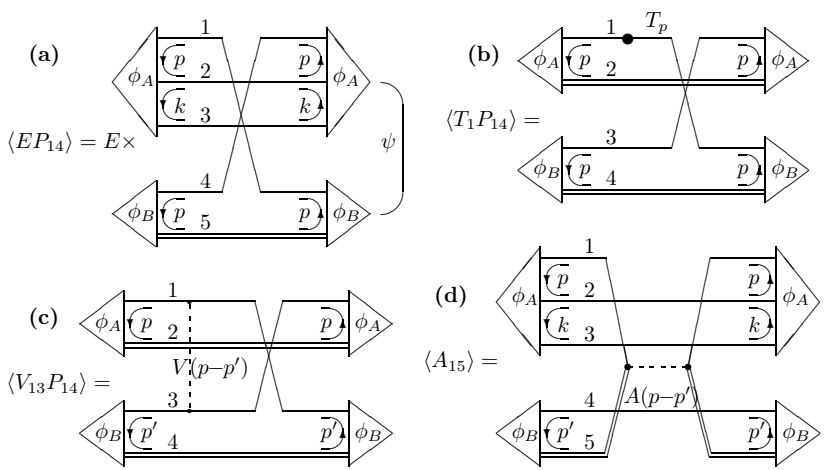

FIGURE 1. We show examples of RGM overlaps, in (a) the norm overlap for the meson-baryon interaction, in (b) a kinetic overlap the meson-meson interaction, in (c) an interaction overlap the mesonmeson interaction, in (d) the annihilation overlap for the meson-baryon interaction.

to show that, in non-exotic channels, the quark-antiquark annihilation could produce a short core attraction. This is confirmed in several studies [8, 9, 10, 13, 14, 15].

The energy of the multiquark is computed with the matrix elements of the hamiltonian,

$$
H=\sum_{i} T_{i}+\sum_{i<j} V_{i j}+\sum_{i \bar{j}} A_{i \bar{j}}
$$

which includes the quark(antiquark) kinetic energies, the quark(antiquark)quark(antiquark) interaction proportional to the colour dependent $\frac{\vec{\lambda}_{i}}{2} \cdot \frac{\vec{\lambda}_{j}}{2}$, and the quark-antiquark annihilation. Once the internal energy of each cluster is accounted, and we also use the antisymmetry of the baryon wave-function, the remaining energy of the meson-meson or baryon-meson system is computed with the overlap,

$$
\begin{aligned}
& \underset{\substack{\text { bar } A \\
\text { mes }}}{\mathscr{C}_{\text {a }}}=3\left\langle\phi_{B} \phi_{A}\right|-\left(V_{14}+V_{15}+2 V_{24}+2 V_{25}\right) P_{14} \\
& +A_{15}\left|\phi_{A} \phi_{B}\right\rangle \\
& \underset{\substack{\text { mesA } A \\
\text { mes } B}}{\mathscr{C}_{\text {m }}}=\left\langle\phi_{B} \phi_{A}\right|\left(1+P_{A B}\right)\left[-\left(V_{13}+V_{23}+V_{14}+V_{24}\right)\right. \\
& \left.\times P_{13}+A_{23}+A_{14}\right]\left|\phi_{A} \phi_{B}\right\rangle \text {, }
\end{aligned}
$$

where we use an obvious notation (see Fig.11). The colour, spin and flavour contributions to the overlaps simply provide an algebraic factor. The momentum (or space) integrations, is estimate them with the variational method to produce a geometrical overlap,

$$
\sum_{s f c} \int_{p} \phi_{A}^{\dagger} \phi_{B}^{\dagger} H \phi_{B} \phi_{A} \simeq M \sum_{s f c} \int_{p} \phi_{A}^{\dagger} \phi_{B}^{\dagger} \phi_{B} \phi_{A}
$$

where $M$ is the sum of hadronic masses corresponding to the Hamiltonian $H$.

When spontaneous chiral symmetry breaking is included in the quark model [8, 16] the annihilation potential turns ou to be crucial [9, 10] to understand the low mass of the $\pi$. The annihilation potential $A$ is also present in the $\pi$ Salpeter equation where it cancels 
most of the kinetic energy and confining potential $2 T+V$. From the quark model with chiral symmetry breaking we get the sum rules [8],

$$
\begin{aligned}
\langle 2 T+V+A\rangle_{S=0} & =M_{\pi} \simeq 0 \\
\langle 2 T+V\rangle_{S=0} & =\frac{2}{3}\left(2 M_{N}-M_{\Delta}\right) \\
\Rightarrow\langle A\rangle_{S=0} & \simeq-\frac{2}{3}\left(2 M_{N}-M_{\Delta}\right) .
\end{aligned}
$$

In eq. (4) the matrix elements are evaluated for s-wave and spin 0 wave-functions of light quarks. Similar sum rules also exist for spin 1 wave-functions, involving only the mass of the $\Delta$ baryon. The annihilation potential for the strange quark mass is smaller by a factor of $\sigma$ which is a power of the constituent quark mass ratio $M_{u, d} / M_{s}$. From eqs. (4) it is clear that the annihilation potential provides an attractive (negative) overlap.

In what concerns the quark-quark(antiquark) potential, in the present case of s-wave states, with a $S=0$ kaon, the only potential which may contribute is the hyperfine potential, proportional to $\frac{\vec{\lambda}_{i}}{2} \cdot \frac{\vec{\lambda}_{j}}{2} \vec{S}_{i} \cdot \vec{S}_{j}$. We find that the total matrix element is a hyperfine splitting,

$$
\left\langle P_{13}\left(V_{13}+V_{23}+V_{14}+V_{24}\right)\right\rangle=4 \frac{2}{3}\left(M_{\Delta}-M_{N}\right)
$$

and it is repulsive (positive).

These results are independent of the particular quark model that we choose to consider, providing it is chiral invariant. We therefore arrive at the criterion - whenever the two interacting hadrons have a common flavour, the repulsion is increased,

- when the two interacting hadrons have a matching quark and antiquark the attraction is enhanced.

In this paper we are interested in the class of resonances which can be understood as a $\mathrm{S}=-1$ kaon $s \bar{u}$ or $s \bar{d}$ trapped by a s-wave hadron. With our criterion we can exclude all hadrons with an antiquark $\bar{u}$ or $\bar{d}$ or with a quark $s$ because the exchange overlap $\left\langle P_{13}\right\rangle$ would be allowed, and this certainly contributes to repulsion. We assume that the attraction is not sufficient to overcome this repulsion. In what concerns attraction we need a quark $u$ or $d$ in the s-wave partner of the kaon, in order to produce annihilation. This excludes the mesons $\eta, \eta^{\prime} \omega, \phi$. Moreover we specialize in systems which are possible to study experimentally, where the kaon partner is a hadronic resonance with a very narrow width. This restricts the kaon partner to s-wave mesons and baryons, and excludes wider resonances like the meson $\rho$ and the baryon $\Delta$. The pion is also excluded because it is too light to bind to the kaon, all that we can get is a very broad resonance, the kappa resonance [17]. Therefore the hadrons which are best candidates to strongly bind the Kaons $s \bar{l}$ are the s-wave hadrons with flavour $l \bar{s}, l \bar{c}, l \bar{b}, l l l, l l c, l l b, l c c, l c b, l b b$. This is expected to result in the $f_{0}(980)$ [17], the $D_{s}\left(2320\right.$ [4] the $D_{s}(2463)$ [1], the $\Lambda(1405)$ and several other predicted resonances.

A convenient basis for the meson and baryon wave-functions is the harmonic oscillator basis, parametrized by the inverse radius $\alpha$. We summarize [9, 10, 15] the effective 
potentials computed for the different channels,

$$
\begin{aligned}
V_{K-K} & =2(1+\sigma)^{2} \frac{1}{6}\langle A\rangle\left|\phi_{000}^{\alpha}\right\rangle\left\langle\phi_{000}^{\alpha}\right|, \\
V_{K-D, D^{*}, B, B^{*}} & =2 \frac{1}{6}\langle A\rangle\left|\phi_{000}^{\alpha}\right\rangle\left\langle\phi_{000}^{\alpha}\right|, \\
V_{K-N} & =4 \frac{1}{6}\langle A\rangle\left|\phi_{000}^{\alpha}\right\rangle\left\langle\phi_{000}^{\alpha}\right|, \\
V_{K-\Sigma_{c}, \Sigma_{b}} & =\frac{7}{3} \frac{1}{6}\langle A\rangle\left|\phi_{000}^{\alpha}\right\rangle\left\langle\phi_{000}^{\alpha}\right|, \\
V_{K-\Xi_{c c}, \Xi_{c b}, \Xi_{b b}} & =2 \frac{1}{6}\langle A\rangle\left|\phi_{000}^{\alpha}\right\rangle\left\langle\phi_{000}^{\alpha}\right|,
\end{aligned}
$$

where the colour and spin factors contribute respectively with $1 / 3$ and $1 / 2,\langle A\rangle$ is of the order of $430 \mathrm{MeV}$ and the geometrical factor provides the separable potential $\left|\phi_{000}^{\alpha}\right\rangle\left\langle\phi_{000}^{\alpha}\right|$. The remaining factor is the flavour factor, the only one that turns out to differ in the s-wave kaon-hadron annihilation. The parameter $\alpha$ is are the only model one, and from the $D_{s}(2320)$ channel we $\alpha=285 \mathrm{MeV}$.

This parametrization hard core interaction in a separable potential, enables us to use standard techniques [15] to exactly compute the scattering $T$ matrix. The binding occurs when the $T$ matrix has a pole for a negative relative energy, and this happens when,

$$
-4 \mu v \geq \frac{\alpha^{3}}{\beta}
$$

The results are displayed in Tables 11 and 2 We conclude that the $f_{0}(980$ and the $D_{s}^{(0+)}, D_{s}^{(1+)}, B_{s}^{(0+)}, B_{s}^{(1+)}$ belong to the same class of tetraquark hadronic resonances. This class is consistent with the picture of a kaon trapped by a s-wave meson. In what concerns pentaquarks, where the kaon is trapped for instance by a nucleon to produce a $\Lambda$, or by other hadrons with $\mathrm{u}$ or $\mathrm{d}$ light quarks, our results predict that we have also have binding with the quantum numbers of the $\Lambda$ and $\Xi_{c}^{+}, \Xi_{c}^{0}, \Xi_{b}^{0}, \Xi_{b}^{-}$and also of the $\Omega_{c c}, \Omega_{c b}$, and $\Omega_{b b}$.

Here we neglected the coupling to the pion-hadron channels. This is correct for the multiquarks of Table1 1 where this coupling is isospin violating. However in the systems of Table 2 where we found a deep binding, we plan to include the coupling to the pionhadron channels. This coupling is expected to decrease substantially the binding energy. We also expect that our method addresses the protonomium recently discovered at BES [18] and the deuson X(3872) recently discovered at BELLE [19].

\section{ACKNOWLEDGMENTS}

We are very grateful to Emilio Ribeiro for discussions on the RGM and to George Rupp for discussions on hadronic resonances. The work of G. Marques is supported by Fundação para a Ciência e a Tecnologia under the grant SFRH/BD/984/2000. 
TABLE 1. This table summarizes the parameters $\mu, v, \alpha, \beta$ and binding energies $B$ (in MeV). The italic binding energy $B_{t h}$ of the $D_{s}(1327)$ is fitted from experiment

\begin{tabular}{c|ccccc} 
channel & $\mu_{\text {exp }}$ & $v_{t h}$ & $\alpha=\beta$ & $B_{t h}$ & $B_{\exp }$ \\
\hline$D_{s}(2317)=\frac{K^{-} \bar{D}^{0}+\bar{K}^{0} D^{-}}{\sqrt{2}}$ & 392 & -143 & 285 & 46 & 46 \\
$D_{s}(2457)=\frac{K^{-} \bar{D}^{* *}+\bar{K}^{0} D^{*-}}{\sqrt{2}}$ & 398 & -143 & 285 & 47 & 46 \\
$\frac{K^{-} \bar{B}^{0}+\bar{K}^{0} B^{-}}{\sqrt{2}}$ & 453 & -143 & 285 & 55 & - \\
$\frac{K^{-} \bar{B}^{* 0}+\bar{K}^{0} B^{*-}}{\sqrt{2}}$ & 454 & -143 & 285 & 55 & - \\
$\frac{K^{-} \Xi_{c c}^{++}+\bar{K}^{0} \Xi_{c c}^{+}}{\sqrt{2}}$ & 442 & -143 & 285 & 53 & - \\
$\frac{K^{-} \Xi_{c b}^{+}+\bar{K}^{0} \Xi_{c b}^{0}}{\sqrt{2}}$ & 466 & -143 & 285 & 56 & - \\
$\frac{K^{-} \Xi_{b b}^{0} \bar{K}^{0} \Xi_{b b}^{-}}{\sqrt{2}}$ & 475 & -143 & 285 & 58 & - \\
\hline
\end{tabular}

TABLE 2. Results for channels open to pion decay.

\begin{tabular}{c|ccccc} 
channel & $\mu_{\exp }$ & $v_{t h}$ & $\alpha=\beta$ & $B_{t h}$ & $B_{\exp }$ \\
\hline$f_{0}(980)=\frac{K^{-} K^{+}+\bar{K}^{0} K^{0}}{\sqrt{2}}$ & 248 & -207 & 285 & 59 & $12 \pm 10$ \\
$\Lambda(1405)=\frac{K^{-} p+\bar{K}^{0} n}{\sqrt{2}}$ & 325 & -286 & 285 & 149 & $30 \pm 4$ \\
$\frac{K^{-} \Sigma_{c}^{++}+\bar{K}^{0} \Sigma_{c}^{+}}{\sqrt{2}}$ & 412 & -167 & 285 & 68 & - \\
$\frac{K^{-} \Sigma_{b}^{+}+\bar{K}^{0} \Sigma_{b}^{0}}{\sqrt{2}}$ & 456 & -167 & 285 & 75 & - \\
\hline
\end{tabular}

\section{REFERENCES}

1. B. Aubert et al. [BABAR Collaboration], Phys. Rev. Lett. 90, 242001 (2003) |arXiv:hep-ex/0304021|.

2. D. Besson et al. [CLEO Collaboration], Phys. Rev. D 68, 032002 (2003) |arXiv:hep-ex/0305100|.

3. P. Krokovny et al. [Belle Collaboration], arXiv:hep-ex/0308019.

4. G. Rupp and E. van Beveren, Phys.Rev.Lett. 91, 012003(2003) |arXiv:hep-ph/0305035|.

5. M.A. Nowak, M. Rho and I. Zahed, Phys. Rev. D48, 4370 (1993) hep-ph/9209272

6. W.A. Bardeen and C. T. Hill, Phys. Rev. D49, 409 (1994) hep-ph/9304265

7. N. Tornqvist, Phys. Rev. Lett. 67, 556 (1991).

8. P. J. Bicudo and J. E. Ribeiro, Phys. Rev. D 42, 1611 (1990); 1625 (1990); 1635 (1990).

9. P. Bicudo, S. Cotanch, F. Llanes-Estrada, P. Maris, E. Ribeiro and A. Szczepaniak, Phys. Rev. D 65, 076008 (2002) |arXiv:hep-ph/0112015|; P. Bicudo, Phys. Rev. C 67, 035201 (2003).

10. P. Bicudo, M. Faria, G. M. Marques and J. E. Ribeiro, arXiv:nucl-th/0106071

11. R. L. Jaffe, Phys. Rev. D 15281 (1977); D. Strottman, Phys. Rev. D 20748 (1979).

12. J. Wheeler, Phys. Rev. 52, 1083 (1937); 52, 1107 (1937).

13. J. E. Ribeiro, Z. Phys. C 5, 27 (1980).

14. J. Dias de Deus and J. Ribeiro Phys. Rev. D 21, 1251 (1980).

15. P. Bicudo and J. E. Ribeiro, Z. Phys. C 38, 453 (1988); P. Bicudo, J. E. Ribeiro and J. Rodrigues, Phys. Rev. C 52, 2144 (1995).

16. P. J. Bicudo, Phys. Rev. C 60, 035209 (1999).

17. E. van Beveren et al., Z. Phys. C 30, 615 (1986).

18. J. Z. Bai et al. [BES Collaboration], Phys. Rev. Lett. 91, 022001 (2003) |arXiv:hep-ex/0303006].

19. K. Abe et al. [Belle Collaboration], arXiv:hep-ex/0308029 